\title{
Enzymes in Organic Chemistry: Transglycosilation
}

\author{
Nenad B. Milosavić*
}

Department of Biochemistry, Faculty of Chemistry, University of Belgrade, Serbia

\begin{abstract}
Therapeutic actions of a significant number of drugs today rely on the presence of carbohydrate moiety. Combinations of carbohydrates and other specific molecules, known as glycosides, comprise a wide range of drug types. To meet these aspects remarkable advances have been made in recent years in exploiting transglycosylation activity for oligosaccharide and glycoconjugate synthesis.
\end{abstract}

The relatively recent recognition of carbohydrates as a medicinally relevant class of biomolecules has led to the investigation or therapeutic agents based on glycan structure or mimics thereof. The development of carbohydrate-based therapies depends on the synthetic accesibility of novel glycoconjugates for study. Unfortunately, decades of synthetic research have yet to provide analogous methods for automated oligosaccharide synthesis. This is the result of carbohydrate structure, and is mainly due to to the fact that even the simplest monosaccharides contain several hydroxyl groups of very similar chemical reactivity. When attempting to construct even a simple oligosaccharide, each hydroxyl group must be distinguished from the others in order to obtain the desired product, with correct regio- and stereochemistry. The hydroxyl differentiation process has generally been tedious, making arduous shuffling of protection group and lengthy synthetic routes that hallmark synthetic carbohydrate chemistry. Chemical synthesis of glycosidic moieties are mainly based on time-consuming protection and deprotection strategies, activation or metal catalysis, but are often accompanied by the formation of unwanted diastereomers and low yields. However, these difficulties can be overcome by the application of enzymatic syntheses. Both glycosyltransferases and glycosidases have been intesively studied for synthetic purposes [1] Transglycosylation reactions are well known and widely used methods for glucoside syntheses [2]. In comparation with glycosyltransferasecatalyzed reaction that use complex sugar nucleotide as the donor and usually has very stringent substrate specificity, glucosidase-catalyzed transglycosylation has several advantages, including the use of readily available donor substrates and the relaxed substrate specificity for acceptors. Nevertheless, the use of glycosidases in synthesis is subject to two major limitations: the low transglycosylation yield and the product hydrolysis. Significant progresses have been made in recent years to overcome these problems. In the last few years some research groups reported transglycosylation reaction with very high glycosylation yield [3]. The onother important discovery in the enzymatic synthesis of glucosides is the invention of glycosynthases, a class of novel glycosidase mutants that can promote glycosidic bond formation when a suitable activated glycosyl donor is provided, but do not hydrolyze the newly formed glycosidic linkage [4].

The synthesis involving enzymatic transgycosylation has emerged as a poweful method for making complex oligosaccharides and glycoconjugates that are valuable for fuctional studies and for biomedical applications as well. The full potential of enzymatic transglycosilation is unfolding in front of us these days.

\section{References}

1. Hancock SM, Vaughan MD, Withers SG (2006) Engineering of glycosidases and glycosyltransferases. Curr Opin Chem Biol 10: 509-519.

2. Veličković D, Dimitrijević A, Bihelović F, Bezbradica D, Jankov R, et al. (2011) A highly efficient diastereoselective synthesis of alpha-isosalicin by maltase from Saccharomyces cerevisiae. Process Biochemistry 46: 1698-1702.

3. Veličković D, Dimitrijević A, Bihelović $F$, Bezbradica D, Knežević-Jugović $Z$ et al. (2012) Novel glycoside of vanillyl alcohol, 4-hydroxy-3-methoxybenzyla-D: -glucopyranoside: study of enzymatic synthesis, in vitro digestion and antioxidant activity. Bioprocess Biosyst Eng 35: 1107-1115.

4. Wang LX, Huang W (2009) Enzymatic transglycosylation for glycoconjugate synthesis. Curr Opin Chem Biol 13: 592-600.
*Corresponding author: Nenad B. Milosavić, Department of Biochemistry, Faculty of Chemistry, University of Belgrade, Studentski trg12-16, 11000 Belgrade, Serbia, E-mail: nenadmil@chem.bg.ac.rs

Received September 25, 2012; Accepted September 27, 2012; Published September 29, 2012

Citation: Milosavić NB (2012) Enzymes in Organic Chemistry: Transglycosilation. J Microbial Biochem Technol 4: xi-xi. doi:10.4172/1948-5948.1000e109

Copyright: (C 2012 Milosavić NB. This is an open-access article distributed unde the terms of the Creative Commons Attribution License, which permits unrestricted use, distribution, and reproduction in any medium, provided the original author and source are credited 